\title{
INNER PRODUCT MODULES ARISING FROM COMPACT AUTOMORPHISM GROUPS OF VON NEUMANN ALGEBRAS
}

\author{
BY \\ WILLIAM L. PASCHKE
}

\begin{abstract}
Let $M$ be a von Neumann algebra of operators on a separable Hilbert space $H$, and $G$ a compact, strong-operator continuous group of -automorphisms of $M$. The action of $G$ on $M$ gives rise to a faithful, ultraweakly continuous conditional expectation of $M$ on the subalgebra $N=\{A \in$ $M: g(A)=A \forall g \in G\}$, which in turn makes $M$ into an inner product module over $N$. The inner product module $M$ may be "completed" to yield a self-dual inner product module $\bar{M}$ over $N$; our most general result states that the $W^{*}$. algebra $A(\bar{M})$ of bounded $N$-module maps of $\bar{M}$ into itself is isomorphic to a restriction of the crossed product $M \times G$ of $M$ by $G$. When $G$ is compact abelian, we give conditions for $A(\bar{M})$ and $M \times G$ to be isomorphic and show, among other things, that if $G$ acts faithfully on $M$, then $M \times G$ is a factor if and only if $N$ is a factor. As an example, we discuss certain compact abelian automorphism groups of group von Neumann algebras.
\end{abstract}

1. Crossed products. Let $G$ be a locally compact group (with left Haar measure $d g$ ) acting as a strong-operator continuous automorphism group on $\boldsymbol{M}$. That is, there is a homomorphism $g \rightarrow g(\cdot)$ of $G$ into the group of *-automorphisms of $M$ such that for each $A \in M$, the map $g \rightarrow g(A)$ of $G$ into $M$ is continuous with respect to the strong-operator topology on $M$. We recall the construction of the crossed product of $M$ by $G$ as set forth by M. Takesaki in [8].

Let $L^{2}(G, H)$ denote the set of functions $\Phi: G \rightarrow H$ satisfying (i) for each $\xi \in H$, the complex-valued function $g \rightarrow(\Phi(g)$, $\xi)$ on $G$ is measurable; and (ii) $\int_{G}\|\Phi(g)\|^{2} d g<\infty . L^{2}(G, H)$ is then a Hilbert space with inner product $(\Phi, \Psi)=\int_{G}(\Phi(g), \Psi(g)) d g$. For each $A \in M$, define a bounded operator $\widetilde{A}$ on $L^{2}(G, H)$ by $(\widetilde{A} \Phi)(g)=g^{-1}(A)(\Phi(g))$; for each $g \in G$, let $L_{g}$ be the unitary operator on $L^{2}(G, H)$ defined by $L_{g} \Phi(h)=\Phi\left(g^{-1} h\right)$. We let $M \times G$, the crossed product of $M$ by $G$, denote the von Neumann algebra of operators on $L^{2}(G, H)$ generated by the operators $\tilde{A}(A \in M)$ and $L_{g}(g \in G)$.

Suppose now that $G$ is compact (with normalized Haar measure $d g$ ). For $A \in M, \xi \in H$, we define the function $A \odot \xi \in L^{2}(G, H)$ by $(A \odot \xi)(g)=$ $g^{-1}(A) \xi$ for $g \in G$. Notice that $\widetilde{B}(A \odot \xi)=B A \odot \xi$ and $L_{g}(A \odot \xi)=g(A) \odot \xi$

Received by the editors March 31, 1975.

AMS (MOS) subject classifications (1970). Primary 46L10; Secondary 22D25, 46H25.

Copyright @ 1976, American Mathematical Society 
$\forall B \in M, g \in G$; hence the closed linear span $M \odot H$ of $\{A \odot \xi: A \in M, \xi \in H\}$ is an invariant subspace of $L^{2}(G, H)$ for $M \times G$. It is also invariant for the commutant $(M \times G)^{\prime}$ of $M \times G$. To see this, take $T \in(M \times G)^{\prime}$. For $\xi \in H$, we have $T(I \odot \xi)=T L_{g}(I \odot \xi)=L_{g} T(I \odot \xi) \forall g \in G$, whence it follows that $T(I \odot \xi)=$ $I \odot \eta$ for some $\eta \in H$. For $A \in M$, we then have $T(A \odot \xi)=T \widetilde{A}(I \odot \xi)=$ $\tilde{A} T(I \odot \xi)=\tilde{A}(I \odot \eta)=A \odot \eta$. We record this observation below.

Proposition 1.2. If $G$ is compact, the projection of $L^{2}(G, H)$ on $M \odot H$ belongs to the center of $M \times G$.

In general, of course, $M \odot H$ may be a proper subspace of $L^{2}(G, H)$. For a simple example, let $M$ be the algebra of complex $2 \times 2$ matrices acting on 2 dimensional Hilbert space $H, G$ the group of unitary $2 \times 2$ matrices with determinant 1 , and let $G$ act as inner automorphisms on $M$ in the obvious way (i.e. $V(A)=V A V^{*}$ for $\left.A \in M, V \in G\right)$. Easy calculations show that for each $\xi \in H$, the map $\Phi \in L^{2}(G, H)$ defined by $\Phi(V)=V^{*} \xi(V \in G)$ is orthogonal to $M \odot H$.

2. Inner product modules. Let $N$ be a von Neumann algebra of operators on $H$, and let $X$ be a right $N$-module with vector space structure compatible with that of $N$. An $N$-valued inner product on $X$ is a conjugate-bilinear map $\langle\cdot, \cdot\rangle$ : $X \times X \rightarrow N$ satisfying

$$
\begin{array}{ll}
\langle x, x\rangle \geqslant 0 & \forall x \in X ; \\
\langle x, x\rangle=0 & \text { only if } x=0 ; \\
\langle x, y\rangle^{*}=\langle y, x\rangle & \forall x, y \in X ; \\
\langle x \cdot A, y\rangle=\langle x, y\rangle A & \forall x, y \in X, A \in N
\end{array}
$$

(where $x \cdot A$ denotes the right action of $A \in N$ on $x \in X$ ). A right $N$-module equipped with an $N$-valued inner product will be called an inner product module over $N$. Notice that if $X$ is an inner product module over $N$, the set $\{\langle x, y\rangle$ : $x, y \in X\}$ spans a two-sided ideal of $N$; we let $\langle\overline{X, X}\rangle$ denote the ultraweak closure of this ideal. It is shown in [3] that $X$ is normed by $\|x\|_{X}=\|\langle x, x\rangle\|^{1 / 2}$ $(x \in X)$. We write $X^{\prime}$ for the set of $N$-module maps from $X$ to $N$ which are bounded with respect to $\|\cdot\|_{X}$. We make $X^{\prime}$ into a right $N$-module by defining scalar multiplication and right action of $N$ on $X^{\prime}$ by $(\lambda \tau)(x)=\bar{\lambda} \cdot(x)$ and

$$
(\tau \cdot A)(x)=A^{*} \tau(x)\left(\lambda \in \mathrm{C}, \tau \in X^{\prime}, x \in X, A \in N\right) .
$$

We shall regard $X$ as a submodule of $X^{\prime}$ by identifying $x \in X$ with the map $\langle;, x\rangle: X \rightarrow N$. We call $X$ self-dual if $X=X^{\prime}$. The module $X^{\prime}$ may be thought of as the "completion" of $X$ in the sense that the inner product on $X$ can be extended to an $N$-valued inner product on $X^{\prime}$ in such a way as to make $X^{\prime}$ self- 
dual, with the extended inner product satisfying $\langle x, \tau\rangle=\tau(x) \forall x \in X, \tau \in X^{\prime}$ (3.2 of [3]).

Suppose now that $Y$ is a self-dual inner product module over $N$. Each bounded module map $T: Y \rightarrow Y$ possesses an adjoint $T^{*}: Y \rightarrow Y$ such that $\left\langle T^{*} x, y\right\rangle=\langle x, T y\rangle \forall x, y \in Y$.

The *-algebra $A(Y)$ consisting of all such $T$ is a $W^{*}$-algebra; a norm-bounded net $\left\{T_{\alpha}\right\}$ in $A(Y)$ converges ultraweakly to $T \in A(Y)$ if and only if $\left\langle T_{\alpha} x, y\right\rangle \rightarrow$ $\langle T x, y\rangle$ ultraweakly in $N \forall x, y \in Y$ (3.10 of [3]). In [4], M. A. Rieffel constructs a faithful normal *-representation of $A(Y)$ on Hilbert space as follows. Let $Y \otimes H$ be the algebraic tensor product of $Y$ with $H$. Define $[\cdot, \cdot]: Y \otimes H$ $\times Y \otimes H \rightarrow \mathrm{C}$ on elementary tensors by $[x \otimes \xi, y \otimes \eta]=(\langle x, y\rangle \xi, \eta)(x, y \in$ $Y, \xi, \eta \in H$ ). Arguing as in 1.7 of [4] (or using 6.1 of [3] to see that for $x_{1}$, $x_{2}, \ldots, x_{n} \in Y$, the $n \times n$ operator matrix $\left[\left\langle x_{i}, x_{j}\right\rangle\right]$ is nonnegative) one shows that $[\because \cdot]$ extends to a well-defined positive semidefinite conjugate-bilinear form on $Y \otimes H$. Let $Z=\{w \in Y \otimes H:[w, w]=0\}$, so $Z$ is a subspace of $Y \otimes H$ and $K_{0}=(Y \otimes H) / Z$ is a pre-Hilbert space with inner product $\left(w_{1}+Z, w_{2}+\right.$ $Z)=\left[w_{1}, w_{2}\right]$.

REMARK 2.1. For $x \in Y, B \in N, \xi \in H$, direct computation shows that $(x \cdot B) \otimes \xi+Z=x \otimes B \xi+Z$.

Let $K$ be the Hilbert space completion of $K_{0}$. For $T \in A(Y)$, define a linear map $\theta_{0}(T): Y \otimes H \rightarrow Y \otimes H$ by

$$
\theta_{0}(T)(x \otimes \xi)=T x \otimes \xi \quad(x \in Y, \xi \in H) .
$$

It is shown in 5.3 of [4] that $\theta_{0}(T)$ induces a bounded linear map $\theta(T): K \rightarrow$ $K$ satisfying $\theta(T)(x \otimes \xi+Z)=T x \otimes \xi+Z$. One checks without difficulty that $\theta$ is a faithful normal *-representation of $A(Y)$ on $K$.

For $x, y \in Y$, define $x \otimes y: Y \rightarrow Y$ by $x \otimes y(w)=x \cdot\langle w, y\rangle(w \in Y)$. One checks that $x \otimes y \in A(Y)$ with $(x \otimes y)^{*}=y \otimes x$ and $T(x \otimes y)=T x \otimes$ $y \forall T \in A(Y)$. It follows that $\{x \otimes y: x, y \in Y\}$ spans a two-sided ideal of $A(Y)$.

Proposition 2.2. If $\overline{\langle Y, Y\rangle}=N$, then $\{x \otimes y: x, y \in Y\}$ spans an ultraweakly dense two-sided ideal of $A(Y)$.

Proof. If the span of $\{x \otimes y: x, y \in Y\}$ is not ultraweakly dense in $A(Y)$, there is a nonzero projection $P \in A(Y)$ such that $P(x \otimes y)=0 \forall x, y \in Y$. We then have $\left\langle P x, x \times y^{\prime}, y\right\rangle=\left\langle P(x \otimes y) y^{\prime}, x\right\rangle=0 \forall x, y, y^{\prime} \in Y$. Since $\overline{\langle Y, Y\rangle}=N$, this forces $\langle P x, x\rangle=0 \forall x \in X$ and hence $P=0$, a contradiction.

Now let $X$ be an inner product module, not necessarily self-dual, over $N$ with $\overline{\langle X, X\rangle}=N$. In the sequel, we will need to know that the set $\{x \otimes y: x, y$ $\in X\}$ spans an ultraweakly dense *-subalgebra of $A\left(X^{\prime}\right)$. (Here, as elsewhere, we are regarding $X$ as a submodule of $X^{\prime}$, so $x \otimes y(\tau)=x:\langle\tau, y\rangle=x \cdot \tau(y)^{*}$ for 
$\tau \in X^{\prime}$.) It is clear that the span of this set is a *-subalgebra of $A\left(X^{\prime}\right)$; unfortunately, the fact that it is ultraweakly dense seems not to follow directly from 2.2 , but rather depends on certain details of the construction of the extended inner product in [3]. We will require the following lemma.

LEMMA 2.3. For each $\tau \in X^{\prime}$, there is a net $\left\{x_{\alpha}\right\}$ in $X$ such that $\left\langle\tau-x_{\alpha}\right.$, $\left.\tau-x_{\alpha}\right\rangle \rightarrow 0$ ultraweakly in $N$.

Proof. Let $s=\left\{f_{1}, f_{2}, \ldots, f_{n}\right\}$ be a finite set of normal positive linear functionals on $N$, and set $f=f_{1}+f_{2}+\cdots+f_{n}$. If we let $Z_{f}=\{x \in X$ : $f(\langle x, x\rangle)=0$, one checks that $Z_{f}$ is a subspace of $X$ and that $X / Z_{f}$ is a pre-Hilbert space with inner product $\left(x+Z_{f}, y+Z_{f}\right)=f(\langle x, y\rangle)$. Since

$$
\tau(x)^{*} \tau(x) \leqslant\|\tau\|^{2}\langle x, x\rangle \quad \forall x \in X
$$

by 2.8 of [3], the map $x+Z_{f} \rightarrow f(\tau(x))$ is a well-defined bounded linear functional on $X / Z_{f}$, so there is a vector $\tau_{f}$ in the Hilbert space completion of $X / Z_{f}$ such that

$$
\left(x+Z_{f}, \tau_{f}\right)=f(\tau(x)) \quad \forall x \in X
$$

Let $x_{s} \in X$ be such that $\left(x_{s}+Z_{f}-\tau_{f}, x_{s}+Z_{f}-\tau_{f}\right) \leqslant 1 / n$. We have $f(\langle\tau, \tau\rangle)$ $=\left(\tau_{f}, \tau_{f}\right)$ by $O_{s}{ }^{h}$ e construction of the extended inner product on $X^{\prime}$ in 3.2 of [3], so $f\left(\left\langle\tau-{ }_{s} \tau-x_{s}\right\rangle\right) \leqslant 1 / n$ and hence $f_{i}\left(\left\langle\tau-x_{s}, \tau-x_{s}\right\rangle\right) \leqslant 1 / n$ for $1 \leqslant i \leqslant$ $n$. It is now clear that the net $\left\{x_{s}\right\}$ (indexed by the inclusion-directed family of finite sets of normal positive linear functionals on $N$ ) has the required property.

Let $\theta: A\left(X^{\prime}\right) \rightarrow B(K)$ (the algebra of bounded operators on $K$ ) be the *. representation of $A\left(X^{\prime}\right)$ on the Hilbert space $K$ constructed at the beginning of this section.

LEMMA 2.4. Let $\tau \in X^{\prime}$ and let $\left\{x_{\alpha}\right\}$ be as in 2.3. Then for each $\xi \in H$, the net $\left\{x_{\alpha} \otimes \xi+Z\right\}$ converges to $\tau \otimes \xi+Z$ in the norm of $K$. Moreover, for $\psi, \psi^{\prime} \in X^{\prime}$, the net $\left\{\psi^{\prime} \otimes \psi\left(x_{\alpha}\right)^{*} \xi+Z\right\}$ converges to $\psi^{\prime} \otimes\langle\psi, \tau\rangle \xi+Z$ weakly in $K$.

Proof. The first statement follows from the identity

$$
\left[\left(\tau-x_{\alpha}\right) \otimes \xi,\left(\tau-x_{\alpha}\right) \otimes \xi\right]=\left(\left\langle\tau-x_{\alpha}, \tau-x_{\alpha}\right\rangle \xi, \xi\right) .
$$

If $\psi \in X^{\prime}$ and $f$ is a normal positive linear functional on $N$, we have

$$
\left|f\left(\left\langle\psi, x_{\alpha}\right\rangle\right)-f(\langle\psi, \tau\rangle)\right| \leqslant f(\langle\psi, \psi\rangle)^{1 / 2} f\left(\left\langle\tau-x_{\alpha}, \tau-x_{\alpha}\right\rangle\right)^{1 / 2},
$$

so $\left\langle\psi, x_{\alpha}\right\rangle=\psi\left(x_{\alpha}\right) * \rightarrow\langle\psi, \tau\rangle$ ultraweakly in $N$ and hence $\psi\left(x_{\alpha}\right) * \xi \rightarrow\langle\psi, \tau\rangle \xi$ weakly in $H$. For $\psi^{\prime} \in X^{\prime}$, the map $\eta \rightarrow \psi^{\prime} \otimes \eta+Z$ is a bounded linear transformation from $H$ into $K$, so $\dot{\psi}^{\prime} \otimes \psi\left(x_{\alpha}\right)^{* \xi}+Z \rightarrow \psi^{\prime} \otimes\langle\psi, \tau\rangle \xi+Z$ weakly in $\boldsymbol{K}$. 
Proposition 2.5. Let $X$ be an inner product module over $N$ such that $\overline{\langle X, X\rangle}=N$. Then $\{x \otimes y: x, y \in X\}$ spans an ultraweakly dense *subalgebra of $A\left(X^{\prime}\right)$.

Proof. By 2.2, it will suffice to show that if $W \in B(K)$ commutes with all of the operators $\theta(x \otimes y)(x, y \in X)$, then $W$ commutes with all of the operators $\theta(\tau \otimes \psi)\left(\tau, \psi \in X^{\prime}\right)$. We first show that $W$ commutes with $\theta(\tau \otimes y)$ $\forall \tau \in X^{\prime}, y \in X$. Let $\left\{x_{\alpha}\right\}$ be a net in $X$ approximating $\tau$ as in 2.3. For $\psi_{1}, \psi_{2}$ $\in X^{\prime}, \xi_{1}, \xi_{2} \in H$, we have

$$
\begin{aligned}
(W \theta & \left.(\tau \otimes y)\left(\psi_{1} \otimes \xi_{1}+Z\right), \psi_{2} \otimes \xi_{2}+Z\right) \\
& \left.=\left(W\left(\tau \cdot \psi_{1}(y)^{*}\right) \otimes \xi_{1}+Z\right), \psi_{2} \otimes \xi_{2}+Z\right) \\
& =\left(W\left(\tau \otimes \psi_{1}(y)^{*} \xi_{1}+Z\right), \psi_{2} \otimes \xi_{2}+Z\right) \quad(\text { by } 2.1) \\
& =\lim _{\alpha}\left(W\left(x_{\alpha} \otimes \psi_{1}(y) * \xi_{1}+Z\right), \psi_{2} \otimes \xi_{2}+Z\right) \quad(\text { by } 2.4) \\
& =\lim _{\alpha}\left(W \theta\left(x_{\alpha} \otimes y\right)\left(\psi_{1} \otimes \xi_{1}+Z\right), \psi_{2} \otimes \xi_{2}+Z\right) \\
& =\lim _{\alpha}\left(\theta\left(x_{\alpha} \otimes y\right) W\left(\psi_{1} \otimes \xi_{1}+Z\right), \psi_{2} \otimes \xi_{2}+Z\right) \\
& =\lim _{\alpha}\left(W\left(\psi_{1} \otimes \xi_{1}+Z\right), \theta\left(y \otimes x_{\alpha}\right)\left(\psi_{2} \otimes \xi_{2}+Z\right)\right) \\
& =\lim _{\alpha}\left(W\left(\psi_{1} \otimes \xi_{1}+Z\right), y \otimes \psi_{2}\left(x_{\alpha}\right) * \xi_{2}+Z\right) \quad(\text { by 2.1) } \\
& =\left(W\left(\psi_{1}+\otimes \xi_{1}+Z\right), y \otimes\left\langle\psi_{2}, \tau\right\rangle \xi_{2}+Z\right) \quad \text { (by 2.4) } \\
& =\left(\theta(\tau \otimes y) W\left(\psi_{1} \otimes \xi_{1}+Z\right), \psi_{2} \otimes \xi_{2}+Z\right) .
\end{aligned}
$$

We conclude that $\theta(\tau \otimes y) W=W \theta(\tau \otimes y) \forall \tau \in X^{\prime}, y \in Y$. In exactly the same way, we can now show that $W$ commutes with all of the operators $\theta(\tau \otimes \psi)$ $\left(\tau, \psi \in X^{\prime}\right)$, which concludes the proof.

In [5], Rieffel investigated the notion of "Morita equivalence" among $W^{*}$. algebras. Roughly speaking, two $W^{*}$-algebras are Morita equivalent if their categories of nondegenerate normal *-representations on Hilbert space are equivalent via a linear functor which is "normal" in an appropriate sense. One of the main results of [5] states that two $W^{*}$-algebras $N_{1}$ and $N_{2}$ are Morita equivalent if and only if there is a self-dual inner product module $Y$ over $N_{1}$ with $\overline{\langle Y, Y\rangle}=$ $N_{1}$ such that $A(Y)$ is isomorphic to $N_{2}$. We conclude this section with a discussion of the relationship between $N$ and $A(Y)$, where $Y$ is a self-dual inner product module over $N$, which may be thought of as giving a characterization of Morita equivalence in terms of tensor products with type I factors.

Let $J$ be an index set, and let $N_{j}$ be a copy of $N$ (regarded as a self-dual inner product module over $N$ ) for each $j \in J$. Form $X=\operatorname{UDS}\left\{N_{j}: j \in J\right\}$ as in $\S 3$ of [3] , so $X$ is a self-dual inner product module over $N$. 
Profosition 2.6. $A(X)$ and $N \otimes B\left(l^{2}(J)\right)$ are isomorphic.

Proof. This follows routinely from 1.22 .14 of [6], where for each $j \in J$ we let $V_{j}$ be the partial isometry in $A(X)$ which takes a $J$-tuple $\left\{B_{k}\right\}_{k \in J} \in X$ to the $J$-tuple with $B_{j}$ in the $j_{0}$-slot and 0 's elsewhere $\left(j_{0} \in J\right.$ fixed).

Proposition 2.7. Let $Y$ be a self-dual inner product module over $N$ such that $\overline{\langle Y, Y\rangle}=N$. There is a Hilbert space $H_{1}$ and a projection $E \in N \otimes B\left(H_{1}\right)$ with central cover $I \otimes I_{H_{1}}$ such that $A(Y)$ and $E\left(N \otimes B\left(H_{1}\right)\right) E$ are isomorphic.

Proof. By 3.12 of [3], there is an index set $J$ and a collection $\left\{P_{j}: j \in J\right\}$ of (not necessarily distinct) nonzero projections in $N$ such that $Y$ and $\operatorname{UDS}\left\{P_{j} N\right.$ : $j \in J$ are isomorphic as inner product modules over $N$. Hence, we may regard $Y$ as a submodule of the inner product module $X$ defined above. Define a projection $E$ in $A(X)$ by $E\left\{B_{j}\right\}_{j \in J}=\left\{P_{j} B_{j}\right\}_{j \in J}$ for $\left\{B_{j}\right\}_{j \in J} \in X ; E$ is the projection of $X$ onto $Y$. It is clear that $A(Y)$ and $E A(X) E$ are isomorphic. If $Q$ is a central projection in $A(X)$ majorizing $E$, then $Q y=y \forall y \in Y$ and hence for $y_{1}$, $y_{2} \in Y$ and $x \in X$, we have $Q x \cdot\left\langle y_{1}, y_{2}\right\rangle=Q\left(x \otimes y_{2}\right)\left(y_{1}\right)=\left(x \otimes y_{2}\right)\left(Q y_{1}\right)$ $=\left(x \otimes y_{2}\right)\left(y_{1}\right)=x \cdot\left\langle y_{1}, y_{2}\right\rangle$. Since $\overline{\langle Y, Y\rangle}=N$, this implies that $Q x=x \forall x$ $\in X$, showing that the central cover of $E$ in $A(X)$ is the identity map. To conclude the proof, take $H_{1}=l^{2}(J)$ and apply 2.6.

3. Compact automorphism groups. In this section, $M$ will be a von Neumann algebra of operators on a separable Hilbert space $H$, and $G$ a compact group acting as a strong-operator continuous group of *-automorphisms of $M$. Let $N$ $=\{A \in M: g(A)=A \forall g \in G\}$ be the fixed-point subalgebra for $G$. Define $\Gamma$ : $M \rightarrow M$ by $\Gamma(A)=\int_{G} g(A) d g$ for $A \in M$. (All integrals of this sort are taken in the sense of $\S 1$ of [1]. For $A \in M, \Gamma(A)$ is the unique operator in $M$. such that $f(\Gamma(A))=\int_{G} f(g(A)) d g$ for all ultraweakly continuous linear functionals $f$ on $M$.) It is clear that $\Gamma$ is an ultraweakly continuous faithful conditional expectation of $M$ on $N$. If we define $\langle\cdot, \cdot\rangle: M \times M \rightarrow N$ by $\langle A, B\rangle=\Gamma\left(B^{*} A\right)$, it follows that $\langle\cdot, \cdot\rangle$ is an $N$-valued inner product on $M$ (viewed as a right $N$ module). In general, $M$ is not complete with respect to the norm obtained from this inner product, much less self-dual, but we can form the self-dual inner product module $\bar{M}$ of bounded $N$-module maps of $M$ into $N$. Here, "bounded" means "bounded with respect to the norm obtained from the inner product". We write $\bar{M}$ instead of $M^{\prime}$ to avoid any possible confusion with the commutant of $M$. By 2.8 of [3], $\bar{M}$ consists precisely of all (complex - ) linear $\tau: M \rightarrow N$ for which there is a real $r \geqslant 0$ such that $\tau(A)^{*} \tau(A) \leqslant r\langle A, A\rangle \forall A \in M$. (For such maps, it turns out that $N$-linearity is automatic.)

Let $K$ be the Hilbert space constructed from the tensor product $\bar{M} \otimes H$ as in $\S 2$, and $\theta$ the associated faithful normal *-representation of $A(\bar{M})$ on $K$. 
By 2.4, the subspace of $K$ spanned by $\{A \otimes \xi+Z: A \in M, \xi \in H\}$ is dense in $K$. For $A_{1}, A_{2}, \ldots, A_{n} \in M$ and $\eta_{1}, \eta_{2}, \ldots, \eta_{n} \in H$, we have

$$
\begin{aligned}
\left\|\sum_{i=1}^{n} A_{i} \otimes \eta_{i}+Z\right\|^{2} & =\sum_{i, j}\left(\left\langle A_{i}, A_{j}\right\rangle \eta_{i}, \eta_{j}\right) \\
& =\sum_{i, j} \int_{G}\left(g\left(A_{j}^{*} A_{i}\right) \eta_{i}, \eta_{j}\right) d g \\
& =\sum_{i, j} \int_{G}\left(g^{-1}\left(A_{i}\right) \eta_{i}, g^{-1}\left(A_{j}\right) \eta_{j}\right) d g \\
& =\left\|\sum_{i=1}^{n} A_{i} \odot \eta_{i}\right\|^{2}
\end{aligned}
$$

We conclude that there is an isometry $V$ of $K$ onto the subspace $M \odot H$ of $L^{2}(G, H)$ defined in $\S 1$ such that $V(A \otimes \eta+Z)=A \odot \eta \forall A \in M, \eta \in H$. If we define $\rho: A(\bar{M}) \rightarrow B(M \odot H)$ by $\rho(T)=V \theta(T) V^{*}$ for $T \in A(\bar{M})$, $\rho$ will be a faithful normal *-representation of $A(\bar{M})$ on $M \odot H$.

THEOREM 3.1. $\rho(A(\bar{M}))=M \times\left. G\right|_{M \odot H}$.

Proof. Notice that if $T \in A(\bar{M})$ is such that $T M \subseteq M$, then $\rho(T)(C \odot \eta)$ $=T(C) \odot \eta \forall C \in M, \eta \in H$. For $A, B \in M$ we have $g\left(B^{*} A^{*} A B\right) \leqslant\|A\|^{2} g\left(B^{*} B\right)$ $\forall g \in G$, so $\langle A B, A B\rangle \leqslant\|A\|^{2}\langle B, B\rangle$. It follows that for each $A \in M$, the map $B \rightarrow A B$ of $M$ into $M$ is a bounded $N$-module map, and hence extends uniquely to a map $m_{A} \in A(\bar{M})$. We have $\rho\left(m_{A}\right)(C \odot \eta)=A C \odot \eta=\widetilde{A}(C \odot \eta) \forall C \in M$, $\eta \in H$, so $\rho\left(m_{A}\right)=\left.\widetilde{A}\right|_{M \odot H}$. Likewise each $g \in G$ gives rise to a map $k_{g} \in$ $A(\bar{M})$ such that $k_{g}(B)=g(B) \forall B \in M$. We have $\rho\left(k_{g}\right)(C \odot \eta)=g(C) \odot \eta=$ $L_{g}(C \odot \eta) \forall C \in M, \eta \in H$, so $\rho\left(k_{g}\right)=\left.L_{g}\right|_{M \odot H}$. We conclude that $M \times\left. G\right|_{M \odot H}$ $\subseteq \rho(A(\bar{M}))$.

For the reverse inclusion, consider the projection $I \otimes I \in A(\bar{M})$. We have $\rho(I \otimes I)(C \odot \eta)=I\langle C, I\rangle \eta=\Gamma(C) \odot \eta=I \odot \Gamma(C) \eta($ since $g(\Gamma(C))=\Gamma(C)$ $\forall g \in G) \forall C \in M, \eta \in H$, whence it follows that $\rho(I \otimes I)$ is the projection of $M \odot H$ onto the closed subspace $I \odot H=\{I \odot \xi: \xi \in H\}$. If $S$ is an operator on $M \odot H$ commuting with $M \times\left. G\right|_{M \odot H}$, then $S(I \odot \xi)=S L_{g}(I \odot \xi)=$ $L_{g} S(I \odot \xi) \forall \xi \in H, g \in G$; we conclude that $S(I \odot H) \subseteq I \odot H$, which shows that $\rho(I \otimes I) \in M \times\left. G\right|_{M \odot H}$. For $A, B, C \in M, \eta \in H$, we compute that

$$
\begin{aligned}
\rho(A \otimes B)(C \odot \eta) & =A\langle C, B\rangle \odot \eta=A \Gamma\left(B^{*} C\right) \odot \eta \\
& =\tilde{A}\left(\Gamma\left(B^{*} C\right) \odot \eta\right)=\tilde{A} \rho\left(I \otimes I \widetilde{B}^{*}(C \odot \eta)\right.
\end{aligned}
$$

Hence, $\rho(A \otimes B) \in M \times\left. G\right|_{M \odot H} \forall A, B \in M$. By 2.5, then, $\rho(A(\bar{M})) \subseteq M \times$ $\left.G\right|_{M \odot H}$, which concludes the proof.

COROLlARY 3.2. Let $G$ be a compact, strong-operator continuous group 
of automorphisms of $M$, with fixed-point algebra $N$. Then $M \times G$ is a factor if and only if $N$ is a factor and $M \odot H=L^{2}(G, H)$.

Proof. This follows from 3.1,1.2, and the fact that Morita equivalent $W^{*}$-algebras have isomorphic centers (8.1 of [5]).

REMARK 3.3. If $M \odot H=L^{2}(G, H)$, then $G$ must act faithfully on $M$, i.e. the homomorphism $g \rightarrow g(\cdot)$ of $G$ into the group of *-automorphisms of $M$ must be injective. (If $g \in G$ is such that $g(A)=A \forall A \in M$, then $L_{g} \Phi=\Phi \forall \Phi$ $\in M \odot H$, which forces $g=e$, the identity of $G$, if $M \odot H=L^{2}(G, H)$.) The example at the end of $\S 1$ shows that it is possible for $G$ to act faithfully on $M$ with $N$ a factor without having $M \odot H=L^{2}(G, H)$.

4. Compact abelian automorphism groups. In this section we impose the additional requirement that the compact group $G$ of automorphisms of $M$ be abelian. One of our main purposes will be to shed some light in the abelian case on the condition $M \odot H=L^{2}(G, H)$ which insures the isomorphism of $A(\bar{M})$ with $M \times G$.

We let $\hat{G}$ denote the dual group of $G$. For each character $\lambda \in \hat{G}$, define a subspace $M_{\lambda}$ of $M$ and a linear map $\Gamma_{\lambda}: M \rightarrow M$ by

$$
\begin{aligned}
M_{\lambda} & =\{A \in M: g(A)=\overline{\lambda(g)} A \quad \forall g \in G\}, \\
\Gamma_{\lambda}(A) & =\int_{G} \lambda(g) g(A) d g \quad(A \in M) .
\end{aligned}
$$

(It is not hard to see that, in the terminology of [1], $M_{\lambda}$ is the spectral subspace of $M$ corresponding to $\{\lambda\} \subseteq \hat{G}$ and that the spectrum of the action of $G$ on $M$ is precisely $\left\{\lambda \in \hat{G}: M_{\lambda} \neq 0\right\}$.)

REMARK 4.1. The following properties of the subspaces $M_{\lambda}$ and the maps $\Gamma_{\lambda}$ are all easily checked:

(i) each $M_{\lambda}$ is a two-sided $N$-module;

(ii) $\langle A, B\rangle=B^{*} A$ for $A, B \in M_{\lambda}$;

(iii) $M_{\lambda}^{*}=M_{\bar{\lambda}}$;

(iv) $\left\langle M_{\lambda_{1}}, M_{\lambda_{2}}\right\rangle=0$ for $\lambda_{1} \neq \lambda_{2}$;

(v) $M_{\lambda_{1}} M_{\lambda_{2}} \subseteq M_{\lambda_{1} \lambda_{2}}$

(vi) $\Gamma_{\lambda}$ is a projection of $M$ onto $M_{\lambda}$ and a bounded $N$-module map;

(vii) $\Gamma_{\lambda_{1}} \Gamma_{\lambda_{2}}=0$ for $\lambda_{1} \neq \lambda_{2}$.

Notice in particular that the subspace of $M$ spanned by the $M_{\lambda}$ 's is a *. subalgebra of $M$.

Proposition 4.2. The subspace spanned by $\left\{M_{\lambda}: \lambda \in \hat{G}\right\}$ is ultraweakly dense in $M$.

Proof. Let $f$ be an ultraweakly continuous linear functional on $M$ such that $f\left(M_{\lambda}\right)=0 \forall \lambda \in \hat{G}$. Take $A \in M$ and define a complex-valued continuous 
function $\phi$ on $G$ by $\phi(g)=f\left(g^{-1}(A)\right)$. For each $\lambda \in \hat{G}$, we have

$$
\begin{aligned}
\hat{\phi}(\lambda) & =\int_{G} \overline{\lambda(g)} f\left(g^{-1}(A)\right) d g \\
& =\int_{G} \lambda(g) f(g(A)) d g=f\left(\Gamma_{\lambda}(A)\right)=0,
\end{aligned}
$$

so $\phi=0$ and in particular $f(A)=0$.

As noted above, each $M_{\lambda}$ is an inner product module over $N$ with $N$-valued inner product $\langle A, B\rangle=B^{*} A\left(A, B \in M_{\lambda}\right)$.

Proposition 4.3. Each $M_{\lambda}$ is self-dual.

Proof. Let $\tau: M_{\lambda} \rightarrow N$ be a bounded $N$-module map and for simplicity assume that $\|\tau\| \leqslant 1$. We must produce an operator $B \in M_{\lambda}$ such that $\tau(A)=$ $B^{*} A \forall A \in M_{\lambda}$. By 2.8 of [3] we have $\tau(A)^{*} \tau(A) \leqslant A^{*} A \forall A \in M_{\lambda}$, and it follows from 6.1 of [3] that for $A_{1}, A_{2}, \ldots, A_{n} \in M_{\lambda}$, the matrices $\left[\tau\left(A_{i}\right)^{*} \tau\left(A_{j}\right)\right]$ and $\left[A_{i}^{*} A_{j}\right]$ in $N_{(n)}$, the $W^{*}$-algebra of $n \times n$ matrices with entries in $N$, satisfy

$$
\left[\tau\left(A_{i}\right)^{*} \tau\left(A_{j}\right)\right] \leqslant\left[A_{i}^{*} A_{j}\right] .
$$

Hence for $\xi_{1}, \xi_{2}, \ldots, \xi_{n} \in H$, we have

$$
\begin{aligned}
\left\|\sum_{i=1}^{n} \tau\left(A_{i}\right) \xi_{i}\right\|^{2} & =\sum_{i, j}\left(\tau\left(A_{i}\right)^{*} \tau\left(A_{j}\right) \xi_{j}, \xi_{i}\right) \\
& \leqslant \sum_{i, j}\left(A_{i}^{*} A_{j} \xi_{j}, \xi_{i}\right)=\left\|\sum_{i=1}^{n} A_{i} \xi_{i}\right\|^{2},
\end{aligned}
$$

so there is a bounded linear map $B_{0}:\left[M_{\lambda} H\right] \rightarrow H$ (where $\left[M_{\lambda} H\right]$ is the closed linear span of $\left.\left\{A \xi: A \in M_{\lambda}, \xi \in H\right\}\right)$ satisfying $B_{0} A \xi=\tau(A) \xi \forall A \in M_{\lambda}, \xi \in H$. Extend $B_{0}$ to a bounded operator on $H$ by setting $B_{0}\left(\left[M_{\lambda} H\right]^{\perp}\right)=0$. Take $T \in$ $M^{\prime}$. Clearly, $\left[M_{\lambda} H\right]$ and $\left[M_{\lambda} H\right]^{\perp}$ are invariant subspaces for $T$, so $T B_{0} \eta=0=$ $B_{0} T \eta$ for $\eta \in\left[M_{\lambda} H\right]^{\perp}$, and

$$
T B_{0} A_{\xi}^{\xi}=T \tau(A) \xi=\tau(A) T \xi=B_{0} A T \xi=B_{0} T A \xi \quad \forall A \in M_{\lambda}, \xi \in H .
$$

We conclude that $B_{0} \in M$. Set $B=\Gamma_{\lambda}\left(B_{0}^{*}\right)$, so $B \in M_{\lambda}$. For $A \in M_{\lambda}$, we have

$$
B^{*} A=\int_{G} \overline{\lambda(g)} g\left(B_{0}\right) A d g=\int_{G} g\left(B_{0} A\right) d g=\tau(A),
$$

since $B_{0} A=\tau(A)$ and $g(\tau(A))=\tau(A) \forall g \in G$.

Form the self-dual inner product module $\operatorname{UDS}\left\{M_{\lambda}: \lambda \in \hat{G}\right\}$ over $N$ as in $\S 3$ of [3].

Proposition 4.4. $\bar{M}$ and $\operatorname{UDS}\left\{M_{\lambda}: \lambda \in \hat{G}\right\}$ are isomorphic as inner product modules over $N$. 
Proof. For each $\tau \in \bar{M}$ and $\lambda \in \hat{G}, 4.3$ gives a unique operator $B(\tau)_{\lambda} \in$ $M_{\lambda}$ such that $\tau(A)=B(\tau)_{\lambda}^{*} A \forall A \in M_{\lambda}$. For $\lambda_{1}, \lambda_{2}, \ldots, \lambda_{n}$ distinct characters in $\hat{G}$, we have

$$
\begin{aligned}
\left\|\sum_{i=1}^{n} B(\tau)_{\lambda_{i}}^{*} B(\tau)_{\lambda_{i}}\right\|^{2} & =\left\|\tau\left(\sum_{i=1}^{n} B(\tau)_{\lambda_{i}}\right)\right\|^{2} \\
& \leqslant\|\tau\|^{2}\left\|\left\langle\sum_{i=1}^{n} B(\tau)_{\lambda_{i}}, \sum_{i=1}^{n} B(\tau)_{\lambda_{i}}\right\rangle\right\| \\
& =\|\tau\|^{2}\left\|\sum_{i=1}^{n} B(\tau)_{\lambda_{i}^{*}}^{*} B(\tau)_{\lambda_{i}}\right\|,
\end{aligned}
$$

the last equality holding by virtue of (ii) and (iv) of 4.1. Hence, $\left\|\Sigma_{i=1}^{n} B(\tau)_{\lambda}^{*} B(\tau)_{\lambda_{i}}\right\| \leqslant\|\tau\|^{2}$. We conclude that the map $\tau \rightarrow\left\{B(\tau)_{\lambda}\right\}_{\lambda \in \hat{G}}$ is a bounded $N$-module map of $\bar{M}$ into $\operatorname{UDS}\left\{M_{\lambda}: \lambda \in \hat{G}\right\}$. If $\tau$ has the form $\langle\cdot, A\rangle$ for some $A \in M$, it is clear that $B(\tau)_{\lambda}=\Gamma_{\lambda}(A) \forall \lambda \in \hat{G}$, so in consequence the $\hat{G}$-tuple $\left\{\Gamma_{\lambda}(A)\right\}_{\lambda \in \hat{G}} \in \operatorname{UDS}\left\{M_{\lambda}: \lambda \in \hat{G}\right\} \forall A \in M_{\lambda}$. Let $f$ be a normal positive linear functional on $N$, and take $A \in M, \tau \in \bar{M}$. Since $\tau(B)^{*} \tau(B) \leqslant\|\tau\|^{2} B^{*} B \forall B$ $\in M$ (and hence $\|\tau(B) \xi\| \leqslant\|\tau\|\|B \xi\| \forall B \in M, \xi \in H$ ), it follows that $\tau$ is continuous with respect to the strong-operator topology of $M$. Hence, the function $\phi$ on $G$ defined by $\phi(g)=f(\tau(g(A)))$ is continuous. We have

$$
\begin{aligned}
\hat{\phi}(\lambda)=\int_{G} \overline{\lambda(g)} f(\tau(g(A))) d g & =f\left(\tau\left(\Gamma_{\bar{\lambda}}(A)\right)\right) \\
& =f\left(B(\tau)_{\bar{\lambda}}^{*} \Gamma_{\bar{\lambda}}(A)\right) \forall \lambda \in \hat{G} .
\end{aligned}
$$

Thus

$$
\begin{aligned}
\sum_{\lambda \in G}|\hat{\phi}(\lambda)| & \leqslant \sum_{\lambda \in \hat{G}} f\left(B(\tau)_{\lambda}^{*} B(\tau)_{\lambda}\right)^{1 / 2} f\left(\Gamma_{\lambda}(A)^{*} \Gamma_{\lambda}(A)\right)^{1 / 2} \\
& \leqslant f\left(\sum_{\lambda \in G} B(\tau)_{\lambda}^{*} B(\tau)_{\lambda}\right)^{1 / 2} f\left(\sum_{\lambda \in G} \Gamma_{\lambda}(A)^{*} \Gamma_{\lambda}(A)\right)^{1 / 2} \\
& <\infty \text { because }\left\{B(\tau)_{\lambda}\right\},\left\{\Gamma_{\lambda}(A)\right\} \in \operatorname{UDS}\left\{M_{\lambda}: \lambda \in \hat{G}\right\} .
\end{aligned}
$$

We conclude that

$$
\begin{aligned}
f(\tau(A)) & =\sum_{\lambda \in \hat{G}} \hat{\phi}(\lambda) \\
& =\sum_{\lambda \in \hat{G}} f\left(B(\tau)_{\lambda}^{*} \Gamma_{\lambda}(A)\right) \\
& =f\left(\left\langle\left\{\Gamma_{\lambda}(A)\right\},\left\{B(\tau)_{\lambda}\right\}\right)\right.
\end{aligned}
$$

This shows that $\tau(A)=\left\langle\left\{\Gamma_{\lambda}(A)\right\},\left\{B(\tau)_{\lambda}\right\}\right\rangle \forall \tau \in \bar{M}, A \in M$ and it follows from the construction of the extended inner product of $\bar{M}$ that $\langle\tau, \psi\rangle=\left\langle\left\{B(\tau)_{\lambda}\right\}\right.$, 
$\{B(\psi)\}\rangle \forall \tau, \psi \in \bar{M}$. Hence the map $\tau \rightarrow\left\{B(\tau)_{\lambda}\right\}$ is an inner product module isomorphism of $\bar{M}$ into $\operatorname{UDS}\left\{M_{\lambda}: \lambda \in \hat{G}\right\}$. But it is clear that if $\left\{B_{\lambda}\right\} \in$ UDS $\left\{M_{\lambda}: \lambda \in \hat{G}\right\}$, then $\tau(A)=\left\langle\left\{\Gamma_{\lambda}(A)\right\},\left\{B_{\lambda}\right\}\right\rangle(A \in M)$ defines a $\tau \in \bar{M}$ such that $B(\tau)_{\lambda}=B_{\lambda} \forall \lambda \in \hat{G}$. This completes the proof.

For $\lambda \in \hat{G}$, let $L\left(M_{\lambda}\right)=\left\{A \in M: A M_{\lambda}=0\right\}$ and let $Q_{\lambda}$ be the leftannihilating projection of $M_{\lambda}$ in $M$, i.e. the projection in $M$ such that $L\left(M_{\lambda}\right)=$ $M Q_{\lambda}$.

Proposition 4.5. $Q_{\lambda}$ is the projection of $H$ on $\left[M_{\lambda} H\right]^{\perp}$ and belongs to the center of $N$.

Proof. Let $P_{\lambda}$ be the projection of $H$ on $\left[M_{\lambda} H\right]$. Since this latter subspace is invariant under $M^{\prime}$, we have $P_{\lambda} \in M$, and $I-P_{\lambda} \in L\left(M_{\lambda}\right)$, so $Q_{\lambda} \geqslant I-$ $P_{\lambda}$. But also $I-Q_{\lambda} \geqslant P_{\lambda}$, since $Q_{\lambda} P_{\lambda}=0$, so $Q_{\lambda}=I-P_{\lambda}$, which proves the first statement. Next, we show that $Q_{\lambda} \in N$. For $A \in M_{\lambda}$ and $g \in G$, we have $g\left(Q_{\lambda}\right) A=\lambda(g) g\left(Q_{\lambda} A\right)=0$, so $g\left(Q_{\lambda}\right) \leqslant Q_{\lambda} \forall g \in G$ and we conclude that $g\left(Q_{\lambda}\right)$ $=Q_{\lambda} \forall g \in G$, i.e. $Q_{\lambda} \in N$. Finally, note that $\left[M_{\lambda} H\right]$ is an invariant subspace for $N$, so $Q_{\lambda}$ lies in the center of $N$.

We can now give a characterization of the condition $M \odot H=L^{2}(G, H)$ when $G$ is compact abelian.

Proposition 4.6. The following are equivalent:

(i) $M \odot H=L^{2}(G, H)$;

(ii) $\left[M_{\lambda} H\right]=H \forall \lambda \in \hat{G}$;

(iii) $L\left(M_{\lambda}\right) \cap$ center $(N)=0 \forall \lambda \in \hat{G}$.

Proof. The equivalence of (ii) and (iii) follows from 3.5; we show that (i) and (ii) are equivalent. Suppose first that (i) holds, and take $\lambda \in \hat{G}$. If $\eta \in$ $\left[M_{\lambda} H\right]^{\perp}$, define $\Phi \in L^{2}(G, H)$ by $\Phi(g)=\lambda(g) \eta(g \in G)$. For $A \in M, \xi \in H$, we have $(A \odot \xi, \Phi)=\int_{G}\left(g^{-1}(A) \xi, \lambda(g) \eta\right) d g=\int_{G}(\lambda(g) g(A) \xi, \eta) d g=\left(\Gamma_{\lambda}(A) \xi, \eta\right)$ $=0$, so $\eta=0$ by (i). Now suppose (ii) holds, and take $\Phi \in(M \odot H)^{\perp}$. Given $\xi \in H$, define $\phi \in L^{2}(G)$ by $\phi(g)=(\Phi(g), \xi)$. We claim that $\hat{\phi}(\lambda)=0 \forall \lambda \in \hat{G}$, which will show that $\Phi=0$. Indeed, for $\lambda \in \hat{G}$ and $\epsilon>0$, (i) gives $A_{1}, A_{2}, \ldots$, $A_{n} \in M_{\lambda}$ and $\xi_{1}, \xi_{2}, \ldots, \xi_{n} \in H$ such that $\left\|\xi-\Sigma_{i=1}^{n} A_{i} \xi_{i}\right\| \leqslant \epsilon$. Hence

$$
\begin{aligned}
|\hat{\phi}(\lambda)| & =\left|\int_{G} \overline{\lambda(g)}(\Phi(g), \xi) d g\right| \\
& \leqslant\left|\int_{G} \overline{\lambda(g)}\left(\Phi(g), \sum_{i=1}^{n} A_{i} \xi_{t}\right) d g\right|+\epsilon\|\Phi\| .
\end{aligned}
$$

For $i=1,2, \ldots, n$, we have 


$$
\begin{aligned}
\int_{G} \overline{\lambda(g)}\left(\Phi(g), A_{i} \xi_{i}\right) d g & =\int_{G}\left(\Phi(g), \lambda(g) A_{i} \xi_{i}\right) d g \\
& =\int_{G}\left(\Phi(g), g^{-1}\left(A_{i}\right) \xi_{i}\right) d g \\
& =\left(\Phi, A_{i} \odot \xi_{i}\right)=0,
\end{aligned}
$$

so $|\hat{\phi}(\lambda)| \leqslant \epsilon\|\Phi\|$. As $\epsilon>0$ was arbitrary, we have $\hat{\phi}(\lambda)=0 \forall \lambda \in \hat{G}$, which completes the proof.

As a corollary, we obtain the following abelian version of 3.2.

COROLLARY 4.7. Let $G$ be a compact abelian strong-operator continuous group of automorphisms of $M$, with fixed-point algebra $N$. Then $M \times G$ is a factor if and only if $N$ is a factor and $G$ acts faithfully on $M$.

Proof. In light of 3.2,3.3 and 4.6, we need only show that if $G$ acts faithfully on $M$, and $N$ is a factor, then $\left[M_{\lambda} H\right]=H \forall \lambda \in \hat{G}$, i.e. $Q_{\lambda}=0 \forall \lambda \in$ $\hat{G}$. Notice that since each $Q_{\lambda}$ lies in the center of $N$, we have $Q_{\lambda}=0$ or $I \forall \lambda \in$ $\hat{G}$. Let $S=\left\{\lambda \in \hat{G}: Q_{\lambda}=0\right\}=\left\{\lambda \in \hat{G}: Q_{\lambda} \neq I\right\}=\left\{\lambda \in \hat{G}: M_{\lambda} \neq 0\right\}$. We claim that $S$ is a subgroup of $\hat{G}$. If $\lambda \in S$, then $\bar{\lambda} \in S$ by (iii) of 4.1 .

If $\lambda_{1}, \lambda_{2} \in S$, then $\left[M_{\lambda_{1}} H\right]=H$ and $\left[M_{\lambda_{2}} H\right]=H$, so $M_{\lambda_{1}} M_{\lambda_{2}} \neq 0$, so $M_{\lambda_{1} \lambda_{2}} \neq 0$ by (v) of 4.1 and hence $\lambda_{1} \lambda_{2} \in S$. To see that $S=\hat{G}$, suppose that $g \in G$ is such that $\lambda(g)=1 \forall \lambda \in S$. For $\lambda \in S$ and $A \in M_{\lambda}$ we have $g(A)=\overline{\lambda(g)} A=A$, and for $\lambda \notin S$, we have $M_{\lambda}=0$. Hence, $g$ fixes each $M_{\lambda}$, so by $4.2, g(A)=A \forall A \in M$. Since $G$ acts faithfully on $M, g$ must be the identity element of $G$. We conclude that $S=\hat{G}$.

Takesaki's duality theorem for crossed products (4.5 of [8]) can be used in connection with 4.7 above in determining what sorts of von Neumann algebras can appear as fixed-point algebras for actions of compact abelian groups on $\boldsymbol{M}$. As a sample application, we will prove the following proposition after establishing a preliminary lemma.

Proposirion 4.8. Suppose that there is a faithful strong-operator continuous action of the circle group $T=\{z \in \mathbf{C}:|z|=1\}$ as automorphisms on $M$ for which the fixed-point algebra $N$ is a type I factor. Then $M$ must be of type I.

The proof of 4.8 requires the following lemma.

LEMMA 4.9. Let $K$ be a separable Hilbert space and let the discrete group $Z$ of integers act as automorphisms on $B(K)$. Then $B(K) \times Z$ is of type $I$.

Proof OF LEMMA. Let $\tau_{n}$ be the automorphism of $B(K)$ corresponding to $n \in Z$. Since all *-automorphisms of $B(K)$ are inner, there is a unitary $U \in B(K)$ such that $\tau_{n}(A)=U^{n} A U^{n} \forall A \in B(K), n \in Z$. For $\phi \in l^{2}(Z), \xi \in K$, define a function $\phi \otimes \xi \in L^{2}(Z, K)$ by $\phi \otimes \xi(n)=\phi(n) U^{n} \xi$. It is easily checked that 
the $\phi \otimes \xi$ 's span a dense subspace of $L^{2}(Z, K)$ and that $\left(\phi_{1} \otimes \xi_{1}, \phi_{2} \otimes \xi_{2}\right)=$ $\left(\phi_{1}, \phi_{2}\right)\left(\xi_{1}, \xi_{2}\right) \forall \phi_{1}, \phi_{2} \in l^{2}(Z), \xi_{1}, \xi_{2} \in K$, so we may identify $L^{2}(Z, K)$ in this way with the Hilbert space tensor product $l^{2}(Z) \otimes K$. For $A \in B(K), \phi \in$ $l^{2}(Z), \xi \in K$, we have

$$
\begin{aligned}
\widetilde{A}(\phi \otimes \xi)(n) & =\tau_{n}^{-1}(A)(\phi \otimes \xi)(n)=\phi(n) U^{-n} A U^{n} U^{-n} \xi \\
& =(\phi \otimes A \xi)(n) \quad(n \in Z),
\end{aligned}
$$

so $\tilde{A}(\phi \otimes \xi)=\phi \otimes A \xi$. If we let $V_{m}$ (for $m \in Z$ ) denote the unitary operator on $l^{2}(Z)$ defined by $\left(V_{m} \phi\right)(n)=\phi(n-m)$, we have

$$
\begin{aligned}
L_{m} \tilde{U}^{-m}(\phi \otimes \xi)(n) & =L_{m}\left(\phi \otimes U^{-m} \xi\right)(n) \\
& =\phi(n-m) U^{m-n} U^{-m} \xi \\
& =\phi(n-m) U^{-n} \xi=\left(V_{m} \phi \otimes \xi\right)(n),
\end{aligned}
$$

so $L_{m} \widetilde{U}^{-m}(\phi \otimes \xi)=V_{m} \phi \otimes \xi$. Since $B(K) \times Z$ is generated by the operators $\widetilde{A}(A \in B(K))$ and $L_{m} \widetilde{U}^{-m}(m \in Z)$, it follows that $B(K) \times Z$ is isomorphic with the tensor product of $B(K)$ by the (abelian) von Neumann algebra of operators on $l^{2}(Z)$ generated by the $V_{m}$ 's. This proves the lemma.

ProOF OF 4.8. We have $M \times T$ isomorphic with $A(\bar{M})$ by 4.6 and 4.7. $M \times T$, being Morita equivalent to the type I factor $N$, must be a type I factor by 2.7 , and hence isomorphic with $B(K)$ for some Hilbert space $K$, which must be separable because $M \times T^{\circ}$ acts on a separable Hilbert space. By the duality result of Takesaki alluded to above, there is an action of $Z$ as automorphisms of $B(K)$ such that $B(K) \times Z$ is isomorphic with $M \otimes B\left(L^{2}(T)\right)$. By the lemma, then, this tensor product must be of type $I$ and hence $M$ is of type $I$.

We remark that it is not clear whether this line of argument can be made to yield an analogous result for arbitrary compact abelian groups acting faithfully on $M$. The problem lies in generalizing Lemma 4.9, where one encounters the difficulty that an arbitrary abelian group of automorphisms of $B(K)$ need not be implemented by a group of unitary operators (although each individual automorphism will of course be inner).

5. A class of examples. In this section we construct a class of examples in which several of the features of the analysis developed above stand out in high relief. As a by-product, we obtain what appears to be a new method for constructing outer automorphisms (and indeed compact abelian groups of outer automorphisms) on many $\mathrm{II}_{1}$-factors.

Let $D$ be a countable discrete group with identity $e$, and let $H$ be the Hilbert space $l^{2}(D)$; elements of $H$ will be written as complex-valued functions on $D$. Let $M=V N(D)$, the von Neumann algebra generated by the left regular 
representation of $D$ on $H$. Notice that for $\xi, \eta \in l^{2}(D)$, the convolution formula

$$
(\xi * \eta)(s)=\sum_{t \in D} \xi(t) \eta\left(t^{-1} s\right)(s \in D)
$$

defines a complex-valued function $\xi * \eta$ on $D$ (which may or may not belong to $\left.l^{2}(D)\right)$. We shall call a function $x \in l^{2}(D)$ a left convolver if $x * \xi \in l^{2}(D) \forall \xi \in$ $l^{2}(D)$ and the operator $\xi \rightarrow x * \xi$ is bounded on $l^{2}(D)$. In II.5 of [7] it is shown that every operator in $M$ has the form $\xi \rightarrow x * \xi$ for some left convolver $x$; it is straightforward to verify that convolution on the left by any left convolver gives an operator in $M$. We shall henceforth identify $M$ with the algebra of left convolvers in $l^{2}(D)$ (with convolution for multiplication and involution defined by $x^{*}(s)=\overline{x\left(s^{-1}\right)}(s \in D)$ ). For $s \in D$, we write $\delta_{s}$ for the function (and left convolver) in $l^{2}(D)$ with value 1 at $s$ and 0 elsewhere.

Let $\lambda$ be a character of $D$, i.e. a homomorphism of $D$ into the circle group $T=\{z \in \mathrm{C}:|z|=1\}$. For $x \in M$, define $a_{\lambda}(x) \in l^{2}(D)$ by $a_{\lambda}(x)(s)=\lambda(s) x(s)$ $(s \in D)$. For $\xi \in l^{2}(D), s \in D$, we have

so

$$
\begin{aligned}
\left(a_{\lambda}(x) * \xi\right)(s) & =\sum_{t \in D} \lambda(t) x(t) \xi\left(t^{-1} s\right) \\
& =\lambda(s) \sum_{t \in D} x \overline{(t) \overline{\lambda\left(t^{-1} s\right) \xi}\left(t^{-1} s\right)} \\
& =\lambda(s)(x *(\bar{\lambda} \xi))(s),
\end{aligned}
$$

$$
a_{\lambda}(x) * \xi=\lambda(x *(\bar{\lambda} \xi)),
$$

which shows that $a_{\lambda}(x) \in M$. Routine computations now show that $a_{\lambda}$ is a *automorphism of $M$.

Proposition 5.1. If $D$ is an infinite-conjugacy-class group (so that $M$ is $a \mathrm{II}_{1}$-factor) and $\lambda$ is a nontrivial character of $D$, then $a_{\lambda}$ is an outer automorphism of $M$.

ProOF. Suppose by way of contradiction that there is a unitary $u \in M$ such that $a_{\lambda}(x)=u * x * u^{*} \forall x \in M$. For $s \in D$, define $\xi_{s} \in l^{2}(D)$ by $\xi_{s}(t)=$ $u\left(s^{-1} t s\right)(t \in D)$. We have

$$
\begin{aligned}
\left(u, \xi_{s}\right) & =\sum_{t \in D} u(t) \overline{u\left(s^{-1} t s\right)} \\
& =\sum_{t \in D} u(t) u^{*}\left(s^{-1} t^{-1} s\right) \\
& =\sum_{t \in D} u(t)\left(\delta_{s} * u^{*}\right)\left(t^{-1} s\right) \\
& =\left(u * \delta_{s} * u^{*}\right)(s)=a_{\lambda}\left(\delta_{s}\right)(s) \\
& =\lambda(s) .
\end{aligned}
$$


Now $u$ is a unit vector in $l^{2}(D)$ (because $u * u^{*}=\delta_{e}$ ) and hence so is $\xi_{s}$. Since $\left|\left(u, \xi_{s}\right)\right|=1$, we conclude that $\xi_{s}$ must be a scalar multiple of $u$, so $|u(t)|=$ $\left|u\left(s^{-1} t s\right)\right| \forall s, t \in D$. By assumption, $a_{\lambda}$ is not the identity automorphism of $D$, so there is a $t_{0} \in D \backslash\{e\}$ such that $u\left(t_{0}\right) \neq 0$. Since the set $\left\{s^{-1} t_{0} s: s \in D\right\}$ is infinite, we have a contradiction.

REMARK 5.2. If $u$ is as in the proof above, we have $a_{\lambda}(u)=u$ and hence $\operatorname{supp}(u) \subseteq \operatorname{ker}(\lambda)$. We can therefore replace the assumption in 5.1 that $D$ be an infinite-conjugacy-class group with the weaker condition that the conjugacy class in $D$ of every $t \in \operatorname{ker}(\lambda) \backslash\{e\}$ be infinite.

In [2] , R. Kallman shows that every outer automorphism of an infinite conjugacy class group induces an outer automorphism of the corresponding $\mathrm{II}_{1}$ factor, and points out that there exist infinite-conjugacy-class groups all of whose automorphisms are inner. The example he mentions is the semidirect product $D=Q_{*} \times Q$ (where $Q$ is the additive group of rationals, $Q_{*}$ is the multiplicative group of nonzero rationals, and multiplication in $D$ is defined by $\left(a_{1}, b_{1}\right)\left(a_{2}, b_{2}\right)=\left(a_{1} a_{2}, a_{1} b_{2}+b_{1}\right)$ for $\left.a_{1}, a_{2} \in Q_{*}, b_{1}, b_{2} \in Q\right)$. Although it has no outer automorphisms, $D$ has an abundance of nontrivial characters, since the abelian group $Q_{*}$ is a homomorphic image of $D$; hence $V N(D)$ has many outer automorphisms. It would be interesting to know whether every infinite-conjugacy-class group with no outer automorphisms must necessarily possess a nontrivial character.

Retuming to the general situation, let $G$ be a compact abelian group and suppose that there exists a homomorphism $s \rightarrow \lambda_{s}$ of $D$ onto $\hat{G}$. We proceed to construct a faithful, strong-operator continuous action of $G$ on $M=V N(D)$. For $g \in G, x \in M$, define $g(x) \in M$ by $g(x)(s)=\overline{\lambda_{s}(g)} x(s)(s \in D)$; it is apparent that $g(\cdot)=a_{\lambda}$, where $\lambda$ is the character of $D$ defined by $\lambda(s)=\overline{\lambda_{s}(g)}$, so $g(\cdot)$ is a *automorphism of $M$. It is equally clear that $g \rightarrow g(\cdot)$ is an isomorphism (injective because $s \rightarrow \lambda_{s}$ is onto) of $G$ into the group of *automorphisms of $M$. To show that the action of $G$ on $M$ is strong-operator continuous, take $s \in D$ and let $\left\{g_{n}\right\}$ be a sequence in $G$ with limit $g \in G$. (Note that $\hat{G}$ must be countable, so the topology on $G$ is metrizable.) For $x \in M$, we have

$$
\begin{aligned}
\left\|g_{n}(x) * \delta_{s}-g(x) * \delta_{s}\right\|^{2} & =\sum_{t \in D}\left|g_{n}(x)\left(t s^{-1}\right)-g(x)\left(t s^{-1}\right)\right| \\
& =\sum_{t \in D}\left|\lambda_{t s^{-1}}\left(g_{n}\right)-\lambda_{t s^{-1}}(g)\right|^{2}\left|x\left(t s^{-1}\right)\right|^{2} .
\end{aligned}
$$

This sum goes to 0 as $n \rightarrow \infty$ because $\lambda_{t s^{-1}}\left(g_{n}\right) \rightarrow \lambda_{t s}-1(g) \forall t \in D$. Since $\left\{\delta_{s}: s \in D\right\}$ spans a dense subset of $l^{2}(D)$, strong-operator continuity follows. The spectral subspaces $M_{\lambda}$ for this action of $G$ on $M$ are immediately identifiable; for each $\lambda \in \hat{G}$, we have $M_{\lambda}=\left\{x \in M: \operatorname{supp}(x) \subseteq\left\{s \in D: \lambda_{s}=\lambda\right\}\right\}$. In particular, 
$N=\left\{x \in M: \operatorname{supp}(x) \subseteq D_{1}\right\}$, where $D_{1}$ is the kernel of the homomorphism $s$ $\rightarrow \lambda_{s}$; thus $N$ is isomorphic with $V N\left(D_{1}\right)$. Notice that for $\lambda \in \hat{G}$ and $s \in D$ such that $\lambda_{s}=\lambda$, we have $M_{\lambda}=\delta_{s} * N$, so $M_{\lambda}$ and $N$ are isomorphic as inner product modules over $N$, and $\left[M_{\lambda} H\right]=H$. By 4.6 and 3.1, $M \times G$ is isomorphic with $A(\bar{M})$, which in turn is isomorphic with $N \otimes B\left(l^{2}(\hat{G})\right)$ by 2.6 .

\section{REFERENCES}

1. W. B. Arveson, On groups of automorphisms of operator algebras, J. Functional Analysis 15 (1974), 217-243. MR 50 \#1016.

2. R. R. Kallman, A generalization of free action, Duke Math. J. 36 (1969), 781-789. MR 41 \#838.

3. W. L. Paschke, Inner product modules over $B^{*}$-algebras, Trans. Amer. Math. Soc. 182 (1973), 443-468. MR 50 \#8087.

4. M. A. Rieffel, Induced representations of $C^{*}$-algebras, Advances in Math. 13 (1974), 176-257. MR 50 \#5489.

5. - Morita equivalence for $C^{*}$-algebras and $W^{*}$-algebras, J. Pure Appl. Algebra 5 (1974), 51-96.

6. S. Sakai, $C^{*}$-algebras and $W^{*}$-algebras, Springer, New York, 1971.

7. J. T. Schwartz, $W^{*}$-algebras, Gordon and Breach, New York, 1967. MR 38 \#547.

8. M. Takesaki, Duality for crossed products and the structure of von Neumann alge. bras of type III, Acta Math. 131 (1973), 249-310.

DEPARTMENT OF MATHEMATICS, UNIVERSITY OF KANSAS, LAWRENCE, KANSAS 66045 